\title{
Origin of Pseudo-Variation in High Resolution Neutron Grating Interferometry
}

\author{
Tobias Neuwirth" ${ }^{1,2, a^{*}}$, Michael Schulz ${ }^{1, b}$ and Peter Böni ${ }^{2, c}$ \\ ${ }^{1}$ Technical University of Munich, Heinz Maier-Leibnitz Zentrum (MLZ), Lichtenbergstr. 1, 85748 \\ Garching, Germany \\ ${ }^{2}$ Technical University of Munich, Department of Physics, Chair for Neutron Scattering (E21), \\ James-Franck-Str. 1, 85748 Garching, Germany \\ a Tobias.Neuwirth@frm2.tum.de, ${ }^{b}$ Michael.Schulz@frm2.tum.de, ${ }^{c}$ Peter.Boeni@frm2.tum.de
}

Keywords: Neutron Imaging; Neutron Grating Interferometry; Quantitative nGI

\begin{abstract}
During neutron grating interferometry measurements with a highly collimated neutron beam a pseudo-variation becomes visible in the acquired data. This pseudo-variation prevents the quantitative analysis of the acquired data, as the measured dark field data depends now on both the ultra-small-angle scattering as well as the properties leading to the pseudo-variation. In the following, the origin of this variation and dependence on the collimation of the neutron beam is explained. It will be shown how by changing the stepped grating in an interferometry scan this variation can be eliminated.
\end{abstract}

\section{Introduction}

Neutron grating interferometry (nGI) is a technique used in neutron imaging allowing to indirectly resolve magnetic and material structures in the range of $100 \mathrm{~nm}$ to $20 \mu \mathrm{m}$ by analyzing the ultra-small-angle scattering off such structures [1, 2, 3]. While resolving these structures is independent of the spatial resolution of the imaging instrument, the ability to locate these structures is still dependent on the spatial resolution. The spatial resolution of the instrument denotes the minimum resolvable structure size and is a combination of the geometric resolution of the instrument (L/D-ratio), the scintillator thickness and the effective pixel size of the detector [4]. In this paper we will investigate the influence of the pinhole size on the signal of the dark field image (DFI) measured with nGI. For this purpose we look at the interaction of a highly collimated neutron beam with the movement of the source grating during an nGI-scan. We will show that in this case two intensity oscillations, one generated by the nGI and one generated solely by the source grating, overlap with each other and generate a periodic pseudo-variation in the nGI-signal. Therefore, a quantitative analysis of the sample parameters becomes difficult. This effect depends strongly on the detailed geometry of the nGI setup. We will concentrate on the case of a highly asymmetric Talbot-Lau interferometer, which is the most common implementation for neutron imaging $[1,5,6]$. Lastly we will show how the problem of pseudovariation can be resolved.

\section{Principles of neutron grating interferometry}

In the following we discuss a strongly asymmetric implementation of a Talbot-Lauinterferometer [1,7] at a neutron imaging beamline. We denote this type of interferometer as a neutron grating interferometer (nGI). It allows to recover information about the transmission (TI), the differential phase shift (DPCI) and the scattering under ultra-small-angles (DFI) of a sample in the neutron beam.

An nGI consists of two absorption gratings $\left(\mathrm{G}_{0}\right)$ and $\left(\mathrm{G}_{2}\right)$ and a phase grating $\left(\mathrm{G}_{1}\right)$ [6]. The phase grating generates a complex interference pattern called the Talbot-carpet. At odd fractional 
Talbot-distances this interference pattern shows a maximum in the contrast of the intensity modulation [8]. Due to the periodicity of the interference pattern in the micrometer range, the interference pattern cannot be directly resolved by a standard neutron imaging detector [9] Hence, the analyzer grating $\mathrm{G}_{2}$ is required which has the same period as the interference pattern. It is positioned at an odd fractional Talbot-distance. The source grating $G_{0}$ is needed to provide the required spatial coherence, without decreasing the neutron flux too much [10].

By a translation $\mathrm{x}_{\mathrm{gi}}$ of one of the gratings perpendicular to the grating lines the intensity at the detector oscillates, as either the minima or maxima of the interference pattern are blocked by grating $\mathrm{G}_{2}$. This intensity oscillation can be approximated by:

$$
I\left(x_{g i}, m, n\right)=a_{0}(m, n)+a_{1}(m, n) \cos \left(\frac{2 \pi x_{g i}}{p_{i}}-\varphi(m, n)\right) .
$$

Here $\mathrm{p}_{\mathrm{i}}$ is the period of the moving grating, $\mathrm{a}_{0}(\mathrm{~m}, \mathrm{n})$ the mean value of the oscillation in pixel $(\mathrm{m}, \mathrm{n}) \mathrm{a}_{1}(\mathrm{~m}, \mathrm{n})$ the amplitude and $\varphi(\mathrm{m}, \mathrm{n})$ the phase. In many cases grating $\mathrm{G}_{0}$ is stepped as it typically has the largest period of the three gratings (typically $\approx 1 \mathrm{~mm}$ ) in the most commonly used highly asymmetric nGI-setups. Furthermore, $\mathrm{G}_{0}$ is typically located relatively close to the pinhole of the imaging instrument in order to fit the nGI setup into the beam line.

To calculate the TI, DPCI and DFI, the values of $\mathrm{a}_{0}, \mathrm{a}_{1}$ and $\varphi$ have to be extracted for every pixel from both a scan without a sample (open beam) and a scan with a sample [11]. Afterwards by normalization of these values to their respective open beam values the TI, DPCI and DFI can be extracted [1].

\section{Origin of pseudo-variation}

In a neutron grating interferometer, the intensity of every pixel changes when performing an nGI-scan. In an ideal interferometer this change depends only on the position of the translated grating with respect to its periodicity. Hence, the intensity on the detector does not vary spatially for a particular position. In a real interferometer, however, small errors in the positioning of the gratings and grating manufacturing imperfections lead to a variation in the intensity on the detector. The nGI data used in this paper has been acquired with the nGI of the ANTARES imaging beamline $[1,9,12]$ at the FRM-II. A detailed description of the used nGI-setup may be found in [1]. In Table 1 the experimental parameters for the different scans are given.

Table 1: Parameters of the scans performed for analysis of the pseudo-variation. $P_{D}$ denotes the pinhole diameter, while the different $L_{i}$ 's denote the distance of the respective components to the pinhole. Above it is also shown which scan parameters have been used in the figures presented in this paper.

\begin{tabular}{cccccccc}
\hline $\begin{array}{c}\mathrm{L} / \mathrm{D}- \\
\text { ratio }\end{array}$ & $\mathrm{P}_{\mathrm{D}}[\mathrm{mm}]$ & $\mathrm{L}_{\mathrm{G} 0}[\mathrm{~m}]$ & $\mathrm{L}_{\mathrm{G} 1}[\mathrm{~m}]$ & $\mathrm{L}_{\mathrm{G} 2}[\mathrm{~m}]$ & $\mathrm{L}_{\text {Det }}[\mathrm{m}]$ & FoV $_{\text {Det }}[\mathrm{mm}]$ & Figure \\
\hline $305 \mid 1219$ & $35.68 \mid 8.92$ & 1.850 & 10.830 & 10.853 & 10.862 & diam. 100 & $1,2,3$ \\
$257 \mid 1031$ & $35.68 \mid 8.92$ & 1.650 & 8.850 & 8.868 & 8.878 & $71 \times 76$ & 4 \\
\hline
\end{tabular}

In Fig. 1(a, b) raw open beam images at four different steps of two different stepping scans of $\mathrm{G}_{0}$ are shown. The scan in (a) was performed at $\mathrm{L} / \mathrm{D}=305$ while for the scan in (b) a higher collimation of $L / D=1219$ was used. In (a) the expected intensity oscillation which is homogeneous over the entire image can be seen. This scan has been performed with a low collimation of $\mathrm{L} / \mathrm{D}=305$. In (b) the four raw open beam images show an additional spatial oscillation, which also moves during the stepping scan. In contrast to the scan in (a), a high 
collimation of $\mathrm{L} / \mathrm{D}=1219$ was chosen for this scan, while all other geometric parameters were kept constant. This spatial oscillation is aligned with the orientation of the source grating. In the following, the intensity variation seen in both scans is called the Talbot-oscillation, while we
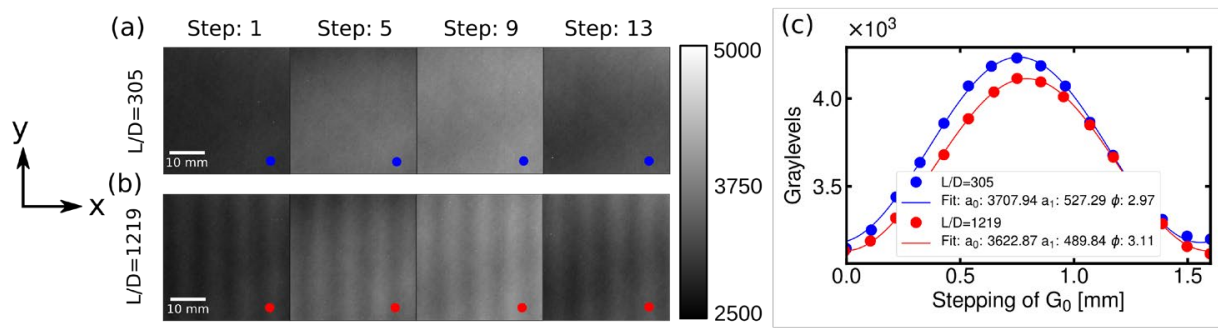

Figure 1: In (a) four steps of a $L / D=305$ and in (b) a $L / D=1219$ scan are shown. The influence of $G_{0}$ is visible in the lower row as a vertical spatial oscillation in the image. In c) the respective curves for the $L / D=1219$ scan (red marker) and the $L / D=305$ scan (blue marker) have been plotted. Decrease of amplitude and mean value of the $L / D=1219$ curve compared to $L / D 305$ curve. This is an effect of the influence of the two oscillations on each other.

denote the rapid oscillation only visible in the high collimation scan as $\mathrm{G}_{0}$-oscillation.

Fig. 1(c) shows the oscillation and the fit parameters of the data of Fig. 1(a), (b) to Eq. 1 for the high collimation (red marker) and low collimation scan (blue marker). The curve of the high collimation scan shows a slightly lower amplitude $\mathrm{a}_{1}$ and mean value $\mathrm{a}_{0}$ than the low collimation scan. This difference is caused by the two overlapping oscillations in the nGI-scan. We have verified that the secondary oscillation is still visible after removing $G_{1}$ and $G_{2}$ from the beamline, proving that $\mathrm{G}_{0}$ must be the cause for the oscillation.

(a)

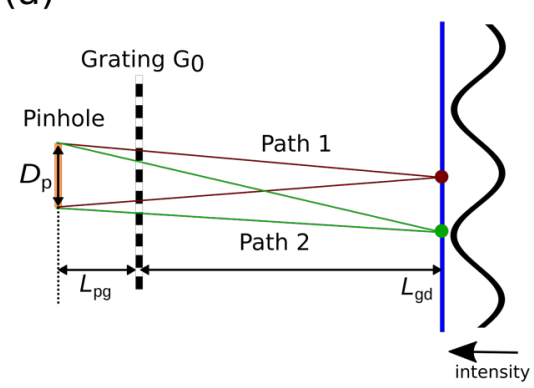

(b)

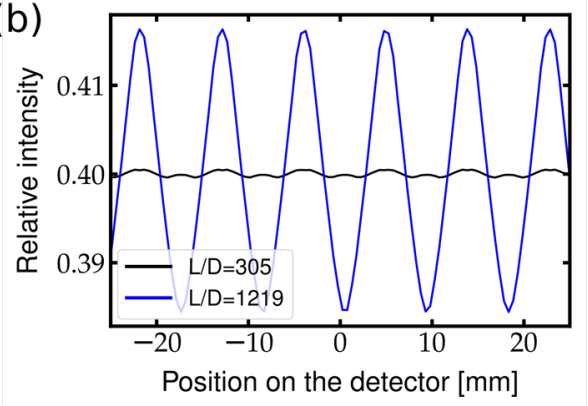

Figure 2: (a) Sketch of the intensity change caused by the introduction of $G_{0}$ into an highly collimated neutron beam. (b) Calculated horizontal intensity curve caused by $G_{0}$ for the $L / D=305$ (black curve) and the $L / D=1219$ (blue curve) at the detector position for an arbitrary vertical position on the detector.

In Fig. 2 an explanation for the oscillation caused by $\mathrm{G}_{0}$ is presented. Panel (a) shows a 2D sketch of the different parts of the pinhole area that are covered by the absorbing lines (black), as seen from different positions on the detector. For this sketch it has been assumed that $G_{0}$ is a binary grating. Path 1 shows the minimum neutron flux possible, while path 2 shows the maximum neutron flux reaching the detector. The number of grating lines illuminated for a point on the detector depends on the chosen pinhole diameter. Consequently also the strength of the variation depends on the pinhole diameter.

In Fig. 2(b), the oscillations on the detector for $\mathrm{L} / \mathrm{D}=305$ and $\mathrm{L} / \mathrm{D}=1219$ have been calculated. For this calculation a binary grating $\mathrm{G}_{0}$ with a duty cycle of 0.4 , a homogeneous neutron beam, a 
distance between pinhole and $\mathrm{G}_{0}\left(\mathrm{~L}_{\mathrm{pg}}=1.95 \mathrm{~m}\right)$ and a distance between $\mathrm{G}_{0}$ and detector $\left(\mathrm{L}_{\mathrm{gd}}=\right.$ $8.92 \mathrm{~m}$ ) were assumed.

Due to the assumption of a homogeneous neutron flux, the visible (non-shadowed) area of the pinhole is directly proportional to the intensity on the detector. In agreement with the duty cycle of 0.4 , the resulting variation has a mean value of $40 \%$ of the maximum intensity. The variation has a period of approximately $9 \mathrm{~mm}$ which is independent of the pinhole diameter. For $\mathrm{L} / \mathrm{D}=1219$ the calculated amplitude relative to the mean value is $3.75 \%$, which is slightly larger than the relative amplitude measured in the $\mathrm{L} / \mathrm{D}=1219$ scan which was around $3.4 \%$. The deviation may be due to the assumption of a perfect $\mathrm{G}_{0}$-grating in the simulation, which is not true for the experiment. Moreover, the effects of the other gratings, being present in the measurement, are neglected. Furthermore, the simulation ignored scattering from the air, which may also contribute to the lower amplitude in the scan. For $\mathrm{L} / \mathrm{D}=305$ the simulation yields an amplitude relative to the mean value of $0.1 \%$. Comparably the noise in the measurement was around $1.5 \%$ of the mean value. Hence, an extraction of the amplitude of the $\mathrm{L} / \mathrm{D}=305$ scan cannot be performed.

In the following, we develop an analytic description of the influence of the $\mathrm{G}_{0}$-oscillation on the acquired nGI-signal. This will allow us to rapidly estimate the influence of the $\mathrm{G}_{0}$-oscillation for different nGI configurations. Due to the round shape of the pinhole, the expected triangular function, which would appear for a square pinhole, is slightly rounded and can be approximated by a cosine function. Similarly to a position change on the detector, a movement of $\mathrm{G}_{0}$ will cause an intensity variation for every pixel dependent on the position of the grating. Therefore the oscillation in one pixel caused by $\mathrm{G}_{0}$ is described similar to Eq. 1:

$$
I^{G 0}\left(x_{g 0}, m, n\right)=a_{0}^{G 0}(m, n)+a_{1}^{G 0}(m, n) \cos \left(\frac{2 \pi x_{g 0}}{p_{0}}-\varphi^{G 0}(m, n)\right) .
$$

$\mathrm{a}_{0}{ }^{\mathrm{G} 0}(\mathrm{~m}, \mathrm{n})$ is the mean value, $\mathrm{a}_{1}{ }^{\mathrm{G} 0}(\mathrm{~m}, \mathrm{n})$ the amplitude and $\varphi^{\mathrm{G} 0}(\mathrm{~m}, \mathrm{n})$ the phase of the $\mathrm{G}_{0^{-}}$ oscillation. The main difference between Eq. 1 and 2 is that $\mathrm{I}^{\mathrm{G} 0}\left(\mathrm{x}_{\mathrm{g} 0}, \mathrm{~m}, \mathrm{n}\right)$ has a periodic variation perpendicular to the $\mathrm{G}_{0}$-grating lines, which is described in the simulation as an oscillation of $\varphi^{\mathrm{G} 0}(\mathrm{~m}, \mathrm{n})$. Grating $\mathrm{G}_{0}$ causes, as shown in Fig. 2 a spatial intensity modulation in the neutron beam which changes the incoming intensity on the other components of the nGI. This incoming intensity is dependent on the stepping position of $\mathrm{G}_{0}$. Hence, to describe the oscillation seen in a pixel, Eq. 1 is modified accordingly:

$$
I^{m o d}\left(x_{g 0}, m, n\right)=I^{G 0}\left(x_{g 0}, m, n\right)+V^{r e a l}(m, n) * I^{G 0}\left(x_{g 0}, m, n\right) \cos \left(\frac{2 \pi x_{g 0}}{p_{0}}-\varphi(m, n)\right)
$$

Here the previously constant offset value $\mathrm{a}_{0}$ has been replaced by the incoming intensity $\mathrm{I}^{\mathrm{G} 0}\left(\mathrm{x}_{\mathrm{g} 0}, \mathrm{~m}, \mathrm{n}\right)$ and $\mathrm{a}_{1}$ has been replaced by $\mathrm{V}^{\text {real }}(\mathrm{m}, \mathrm{n}) * \mathrm{I}^{\mathrm{G} 0}\left(\mathrm{x}_{\mathrm{g} 0}, \mathrm{~m}, \mathrm{n}\right)$. $\mathrm{V}^{\text {real }}(\mathrm{m}, \mathrm{n})$ is the intrinsic visibility which describes the quality of the gratings used, the used setup parameters and the amount of scattering inside the sample.

\section{Comparison of simulated and measured data}

Using Eq. 3 and the parameters extracted from the $\mathrm{L} / \mathrm{D}=1219$ scan, a data set has been simulated and compared with the $\mathrm{L} / \mathrm{D}=1219$ scan. The extracted parameters were $\mathrm{a}_{0}{ }^{\mathrm{G} 0}=2600, \mathrm{a}_{1}{ }^{\mathrm{G} 0}=78$, $\mathrm{V}^{\text {real }}=0.19$ and $(\mathrm{m}, \mathrm{n})=0 . \varphi^{\mathrm{G} 0}$ has a periodic variation over the image as caused by grating $\mathrm{G}_{0}$. In Fig. 3 , the $\mathrm{a}_{0}$-map, the $\mathrm{a}_{1}$-map and the visibility-map of the simulation (a-c) and the $\mathrm{L} / \mathrm{D}=1219$ measurement (d-f) are shown. 
The $\mathrm{a}_{0}$-map ( $\mathrm{a}_{1}$-map) is the image representation of the mean value $\mathrm{a}_{0}$ (amplitude $\mathrm{a}_{1}$ ) evaluated for every pixel. Accordingly, the visibility-map is the pixel-wise division of the $\mathrm{a}_{1}$-map by the $\mathrm{a}_{0}$ map. Table 2 lists the standard deviations of the simulated and measured maps for the areas marked by the red contour in Fig. 3 .

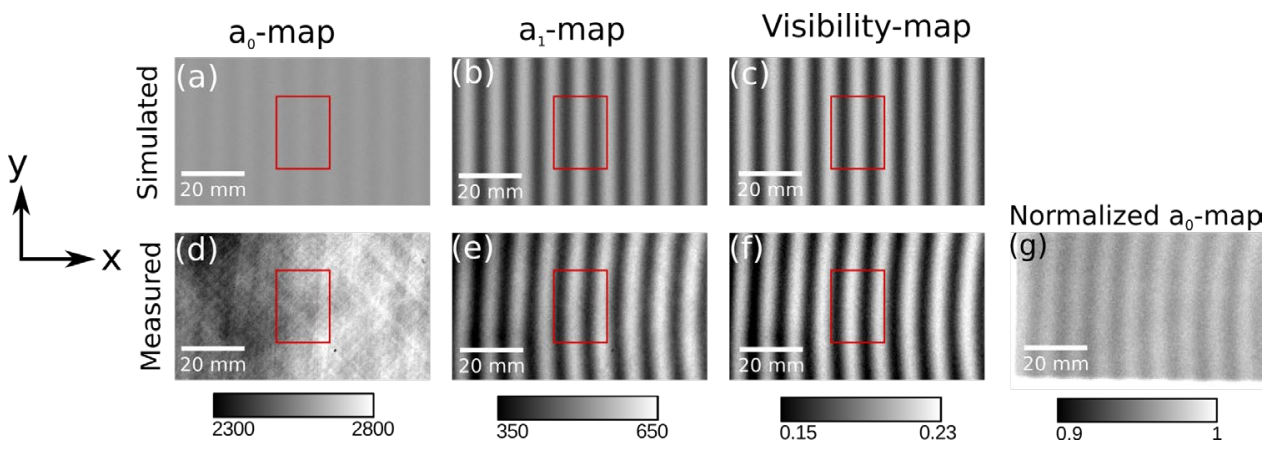

Figure 3: (a-f) Comparison of the $a_{0}$-map, the $a_{1}$-map and the visibility-map simulated using the parameters extracted from the $L / D=1219$ scan and the measured data from the $L / D=1219$ scan.

The $a_{1}$-map and the visibility-map show good agreement of simulation and measurement. In

Table 2, the standard deviations of the areas marked by the red contours are presented. The bending of the stripes in the measurement are caused by non-constant phase of the Talbotoscillation over the image. For the simulation the phase of the Talbot-oscillation has been chosen to be constant. In the measured $a_{0}$-map the periodic variation is strongly suppressed compared to the simulation map, due to inhomogeneities in the neutron beam and the scintillator. In $(g)$ the discernibility of the structure is enhanced by normalizing the $L / D=1219 a_{0}$-map to the $a_{0}$-map of the $L / D=305$ scan.

Table 2: Standard deviation inside the red contour marked in Fig. 3.

\begin{tabular}{cccc}
\hline Standard deviation [arb. unit.] & $\mathrm{a}_{0}$-map & $\mathrm{a}_{1}$-map & visibility-map \\
\hline Simulated & 14 & 52 & 0.020 \\
Measured & 77 & 64 & 0.023 \\
\hline
\end{tabular}

While the standard deviations for the $\mathrm{a}_{1}$-map and the visibility-map are in a similar range, the standard deviation for the $\mathrm{a}_{0}$-map differs strongly. The standard deviations for the measured maps are constantly higher than the simulated maps. This is caused by neglecting the effects of the scintillator structure and the beam inhomogeneity in the simulation. The $\mathrm{a}_{0}$-map of the simulation shows the periodic pseudo-structure while in the $\mathrm{a}_{0}$-map of the measurement this periodic variation is strongly suppressed. This suppression is caused by the inhomogeneity of the measured $\mathrm{a}_{0}$-map, which is considerably stronger than the structure seen in the simulated map.

The inhomogeneity is again an effect of the scintillator structure and the beam inhomogeneity. To effectively visualize the structure in the measured $\mathrm{a}_{0}$-map, it is normalized to the $\mathrm{a}_{0}$-map of the $\mathrm{L} / \mathrm{D}=305$ measurement. This normalization eliminates the inhomogeneities caused by the scintillator structure and the general beam inhomogeneity. In Fig. 3 (g), the resulting image is shown. In this image, it can be clearly seen that the pseudo-structure is also visible in the mean value of the oscillation. Therefore, the TI is also affected by the pseudo-structure, but the effect is suppressed by the general inhomogeneity of the neutron beam. 
Lastly, the simulation shows a perfectly vertical pseudo-structure while the structure in the measurement is slightly bent. From Eq. 3 it follows that the resulting variation depends on the relative phase shift of the $\mathrm{G}_{0}$-oscillation and the Talbot-oscillation. Due to a Moiré-stripe, caused by imperfections in the setup, the phase of the Talbot-oscillation is not static but varies over the image, causing the bending of the resulting pseudo-structure. The direction of this bending depends on the specific form of the Moiré-stripe, which in turn depends on the alignment of the gratings.

\section{Elimination of the pseudo-variation}

As shown above the main problem of the nGI-technique using a tight collimation is the simultaneous occurrence of two intensity oscillations, which both depend on the position of the source grating $G_{0}$. Hence, by stepping either grating $G_{1}$ or $G_{2}$ instead of $G_{0}$ the $G_{0}$-oscillation changes to a static intensity variation that can be removed via normalization. We expect a similar problem of two simultaneous oscillations to occur if $\mathrm{G}_{2}$ is stepped, as it is also an absorption
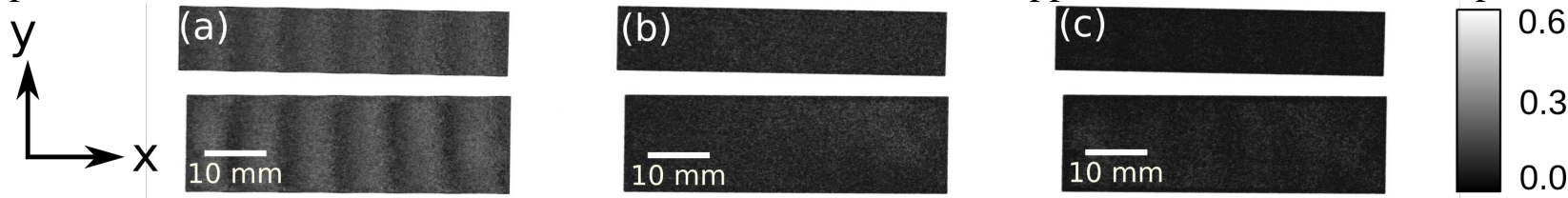

Figure 4: Comparison of the DFI of (a) the $G_{0}-L / D=1031$ scan, with (b) the $G_{1}-L / D=1031$ scan and (c) the reference $G_{0}-L / D=257$ scan.

grating. Hence, $\mathrm{G}_{1}$ has been chosen to be stepped, as it does not produce an intrinsic intensity modulation, but rather a phase modulation. To verify the validity of this approach three nGIscans with different collimations and stepping methods were performed. Four electrical steel sheets were used as test samples.

In Fig. 4, the DFI (a) of a high collimation nGI-scan with $\mathrm{G}_{0}$ stepping $\left(\mathrm{G}_{0}-\mathrm{L} / \mathrm{D}=1031\right)$ is presented and compared to the DFI (b) when stepping $\mathrm{G}_{1}\left(\mathrm{G}_{1}-\mathrm{L} / \mathrm{D}=1031\right)$. Additionally, in (c) a reference scan performed by stepping $G_{0}$ at low collimation is presented $\left(G_{0}-L / D=257\right)$. This scan has been chosen as the reference scan as this is the standard type of nGI-scan performed. For this type of scan the oscillation introduced by $\mathrm{G}_{0}$ is below the noise level and does not affect the scan. It can immediately be seen that the pseudo variation visible in the $G_{0}-L / D=1031$ scan is eliminated in the $\mathrm{G}_{1}-\mathrm{L} / \mathrm{D}=1031$ scan. Additionally, the $\mathrm{G}_{0}-\mathrm{L} / \mathrm{D}=1031$ shows a drift in the setup between the sample and open beam scan, as the pseudo-variation is even visible in the open beam area. Hence, to allow for quantitative measurements with high spatial resolution the stepping of the $G_{1}$ grating is the most promising approach.

\section{Conclusion}

We have shown that nGI measurements with a highly collimated neutron beam can result in a periodic pattern appearing in the visibility and phase map, caused by a projection of the $\mathrm{G}_{0}$ grating. Due to the movement of $\mathrm{G}_{0}$ during an nGI-scan this periodic pattern is not static, but also varies during the scan. This variation combined with the Talbot-oscillation causes an emergence of a pseudo-variation of the visibility-map. In contrast to the Talbot-oscillation, the $\mathrm{G}_{0}$-oscillation is unaffected by ultra-small-angle scattering and refraction of neutrons passing through the sample, which leads to a corruption of the evaluated signal. We have shown that the combination of the $\mathrm{G}_{0}$-oscillation and the Talbot-oscillation experiences a phase shift inside the sample, which in turn leads to the pseudo-structure inside the sample in the DFI.

In order to effectively perform quantitative nGI measurements with high collimation, the stepping scan has been performed by stepping $G_{1}$ instead of $G_{0}$. As a result the periodic pattern 
generated by $\mathrm{G}_{0}$ becomes static and can be easily dealt with by the standard normalization procedure used during data evaluation.

\section{References}

[1] T. Reimann et al. "The new neutron grating interferometer at the ANTARES beamline: design, principles and applications”. In: Journal of Applied Crystallography 49.5 (2016), pp. 1488-1500. https://doi.org/10.1107/S1600576716011080

[2] T. Reimann et al. "Visualizing the morphology of vortex lattice domains in a bulk type-II superconductor”. In: Nature communications 6 (2015), p. 8813. https://doi.org/10.1038/ncomms9813

[3] C. Grünzweig et al. "Bulk magnetic domain structures visualized by neutron dark-field imaging”. In: Applied Physics Letters 93.11 (2008), p. 112504. https://doi.org/10.1063/1.2975848

[4] I. S. Anderson et al. Neutron Imaging and Applications: A Reference for the Imaging Community 2009. Tech. rep. ISBN 978-0-387-78692-6.

[5] Y. Seki et al. "Development of Multi-colored Neutron Talbot-Lau Interferometer with Absorption Grating Fabricated by Imprinting Method of Metallic Glass”. In: Journal of the Physical Society of Japan 86.4 (2017), p. 044001. https://doi.org/10.7566/JPSJ.86.044001

[6] C. Grünzweig et al. "Design, fabrication, and characterization of diffraction gratings for neutron phase contrast imaging”. In: Review of Scientific Instruments 79.5 (2008), p. 053703. https://doi.org/10.1063/1.2930866

[7] E. Lau. “Interference phenomenon on double gratings”. In: Ann. Phys. 6 (1948), p. 417. https://doi.org/10.1002/andp.19484370709

[8] A. Hipp et al. "Energy-resolved visibility analysis of grating interferometers operated at polychromatic X-ray sources”. In: Opt. Express 22.25 (Dec. 2014), pp. 30394-30409. https://doi.org/10.1364/OE.22.030394

[9] M. Schulz et al. “ANTARES: Cold neutron radiography and tomography facility”. In: Journal of large-scale research facilities JLSRF 1 (2015), p. 17. https://doi.org/10.17815/jlsrf-1-42

[10] F. Pfeiffer et al. "Neutron phase imaging and tomography”. In: Physical Review Letters 96.21 (2006), p. 215505. https://doi.org/10.1103/PhysRevLett.96.215505

[11] S. Marathe et al. "Improved algorithm for processing grating-based phase contrast interferometry image sets”. In: Review of Scientific Instruments85.1 (2014), p. 013704. https://doi.org/10.1063/1.4861199

[12] E. Calzada et al. "New design for the ANTARES-II facility for neutron imaging at FRM II". In: Nuclear Instruments and Methods in Physics Research Section A: Accelerators, Spectrometers, Detectors and Associated Equipment 605.1 (2009), pp. 50-53. https://doi.org/10.1016/j.nima.2009.01.192 